\title{
Adubação foliar e via substrato no desenvolvimento inicial de mudas de bromélia imperial ${ }^{(1)}$
}

\author{
DANIELLA NOGUEIRA MORAES CARNEIRO(2), PATRÍCIA DUARTE DE OLIVEIRA PAIVA(3), \\ ÉRICA RESENDE ANDRADE SANTA CECÍLIA ${ }^{(4)}$, LEANDRO FLÁVIO CARNEIRO ${ }^{(5)}$ e RENATO PAIVA ${ }^{(6)}$
}

\begin{abstract}
RESUMO
As bromélias têm sido muito utilizadas em projetos paisagísticos, por sua beleza e resistência, destacando-se a bromélia-imperial (Alcantarea imperialis), espécie bastante usada em paisagismo. Apesar da importância dessas espécies ornamentais, o conhecimento sobre adubação ainda é restrito. O presente trabalho teve como objetivo avaliar o efeito da adubação com boro e zinco em substrato e via foliar sobre o desenvolvimento de mudas de bromélia-imperial. As mudas foram plantadas em vasos de $1 \mathrm{dm} 3$ contendo substrato composto por casca de arroz carbonizada (50\%) e terra de subsuperfície (50\%). Foram testados 8 tratamentos: presença e ausência de adubação via substrato, adubação foliar com boro (1g L ${ }^{-1}$ de $\left.\mathrm{H}_{2} \mathrm{BO} 3\right)$, zinco (1g L $\mathrm{L}^{-1}$ de $\mathrm{ZnSO}_{4}$ ), boro associado ao zinco (nas mesmas concentrações) além da testemunha, constituindo fatorial $2 \mathrm{x} 4$. O experimento foi instalado em delineamento inteiramente casualizado, com 4 repetições. As avaliações foram realizadas sete meses após a implantação do experimento observando-se o incremento de altura, número de folhas por planta, largura das folhas e diâmetro médio das rosetas. Observou-se que o fornecimento de $\mathrm{B}+\mathrm{Zn}$ proporcionou menor incremento na altura e largura das folhas. Menor número de folhas formadas nas mudas foi constatado quando as adubações com $\mathrm{B}$ ou $\mathrm{B}+\mathrm{Zn}$ foram realizadas, não havendo diferenças entre elas. Menor diâmetro da roseta foi observado em plantas cultivadas com adubação via substrato. Observou-se também a ocorrência de necrose nas pontas das folhas das bromélias em conseqüência da adubação foliar. Verificou-se que as plantas tratadas com boro apresentaram maior incidência dessas necroses nas folhas.
\end{abstract}

Palavras-chave: Bromeliaceae, Alcantarea imperialis, boro, zinco.

\section{ABSTRACT \\ Leaf and substrate feeding of Imperial Bromeliad plantlets'initial development}

Bromeliads have been used in landscaping projects, for their beauty and strength, meanly the imperial bromeliad (Alcantarea imperialis), specie most used in gardening. Despite of the importance of these ornamental species, the knowledge of fertilization is still restricted. This study aimed to evaluate the application effect of fertilization with boron and zinc in substrate and on the leaves on the development of seedlings of imperial bromeliad. The seedlings were planted in pots of $1 \mathrm{dm} 3$ containing substrate composed of rice husk carbonized (50\%) and land of subsurface (50\%). Eight treatments were tested: presence and absence of fertilization of substrate and foliar fertilization with boron $\left(1 \mathrm{~g} . \mathrm{L}^{-1} \mathrm{H}_{2} \mathrm{BO}_{3}\right)$, zinc $\left(1 \mathrm{~g}\right.$. L $\left.\mathrm{L}^{-1} \mathrm{ZnSO}_{4}\right)$, boron associated with the zinc (in the same concentrations) plus the witness, constituting in a factorial $2 \times 4$. The experiment was installed on completely randomized design, with 4 replications. The evaluations were conducted seven months after the implementation of the experiment and considered the increase in height, number of leaves per plant, leaves and rosettes diameters. It was observed that the supply of $\mathrm{B}+\mathrm{Zn}$ provided less increase in height and width of the leaves. The lower number of leaves in seedlings occurred when the fertilization with B or B $+\mathrm{Zn}$ were provide, with no differences between them. The worst value of rosette diameter occurred when plants were cultivated with fertilization of substrate. It was also observed the occurrence of necrosis in the tips of the leaves of bromeliads as a result of fertilization leaf. It was found that the plants treated with boron showed a higher incidence of necrosis on leaves.

Keywords: Bromeliaceae, Alcantarea imperialis, boron, zinc.

\footnotetext{
(1) Recebido para publicação em 05/05/2008 e aceito em 17/12/2008

(2) Mestranda em Fitotecnia, DAG, Universidade Federal de Lavras (UFLA) Lavras (MG); daninog27@yahoo.com.br

(3) D. Sc., Prof ${ }^{a}$. Adjunta, DAG/UFLA, C. P. 3037, 37200-000, Lavras (MG).

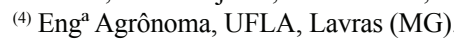

${ }^{(5)}$ M. Sc., Doutorando em Solos e Nutrição de Plantas, DCS/UFLA, Lavras (MG).

(6) Ph. D., Prof. Adjunto, DBI /UFLA, Lavras (MG)
} 


\section{INTRODUÇÃO}

As bromélias têm sido muito utilizadas em projetos paisagísticos, devido à sua beleza, resistência e praticidade no manuseio, o que contribui para sua importância econômica. Ainda são consideradas verdadeiras amplificadoras da biodiversidade local, pois acumulam água entre suas bainhas, proporcionando o desenvolvimento de microorganismos, insetos e ainda, permitindo a alimentação de pequenos animais (CARVALHO, 1997). Apesar desse uso, a produção em larga escala ainda é pequena, o que faz com que sejam comercializadas plantas retiradas do seu habitat (MELO, 1996).

Dentre as espécies mais usadas em paisagismo, pode-se destacar a Alcantarea imperialis V.L.Harms, originária das escarpas rochosas íngremes da Serra dos Órgãos, RJ (SMITH \& DOWNS, 1977). Essa espécie possui um desenvolvimento lento, conseqüência da pequena disponibilidade de nutrientes nesse ecossistema. Em produção comercial, os nutrientes são supridos com adubação, o que diminui seu ciclo consideravelmente (LEME \& MARIGO, 1993). Contudo, as deficiências de B (Boro) e Zn (Zinco) são as mais frequentes e pouco se sabe sobre doses, modos eficientes de aplicação e critérios seguros para o diagnóstico da necessidade de adubação com esses nutrientes (QUAGGIO \& PIZA JUNIOR, 2001).

As Bromeliáceas absorvem nutrientes com facilidade pelos tricomas, localizados na base das folhas, o que viabiliza a utilização de adubos foliares, a fim de acelerar o crescimento da planta (PAULA, 2000). Essa efetividade de adubação foliar foi observada por FERREIRA et al (2007) em cultivo de Neoregélia cruenta. RODRIGUES (2004) relata que a bromélia imperial responde bem à adubação durante a fase de desenvolvimento das mudas, sugerindo sua aplicação na formulação desenvolvida por MALAVOLTA (1980).

PAULA (2000), por outro lado, recomenda evitar a aplicação de $\mathrm{B}$, pois esse nutriente provoca queimaduras na ponta das folhas, o que também acontece com o uso em excesso de $\mathrm{P}$ (fósforo). Apesar de não haver registro, produtores de mudas de bromélias sugerem que o uso de $\mathrm{Cu}$ (Cobre) e $\mathrm{Zn}$ pode ser fitotóxico às bromélias, mesmos quando aplicados em pequenas doses.

$\mathrm{O}$ presente trabalho teve como objetivo avaliar o efeito da adubação com boro (B) e zinco ( $\mathrm{Zn}$ ) em substrato e via foliar sobre o desenvolvimento de mudas de Alcantarea imperialis V.L.Harms.

\section{MATERIAL E MÉTODOS}

Utilizaram-se mudas de bromélia Alcantarea imperialis V.L.Harms, produzidas em laboratório de cultura de tecidos. Após a aclimatização, as mudas, medindo cerca de 7 centímetros, foram transferidas para vasos plásticos de 1 dm3 - uma muda por vaso - contendo substrato composto por casca de arroz carbonizada (50\%) e terra de subsuperfície $(50 \%)$, conforme recomendação de RODRIGUES et al. (2004).

$\mathrm{O}$ experimento foi instalado em delineamento inteiramente casualizado, com 4 repetições. Os tratamentos foram desenvolvidos em fatorial $2 \times 4$ (tabela 1), sendo: T1: Sem adubação (testemunha); T2: Aplicação de $1 \mathrm{~g}$. $\mathrm{L}^{-1}$ de boro via foliar $\left(\mathrm{H}_{3} \mathrm{BO}_{4}\right)$; T3: 1g. $\mathrm{L}^{-1}$ de zinco via foliar $\left(\mathrm{ZnSO}_{4}\right)$; T4: 1g. $\mathrm{L}^{-1}$ boro $\left(\mathrm{H}_{3} \mathrm{BO}_{4}\right)+1 \mathrm{~g}$. $\mathrm{L}^{-1}$ zinco $\left(\mathrm{ZnSO}_{4}\right)$ via foliar; T5: adubação no substrato; $\mathrm{T} 6$ : adubação no substrato $+1 \mathrm{~g}$. $\mathrm{L}^{-1}$ de boro via foliar $\left(\mathrm{H}_{3} \mathrm{BO}_{4}\right)$; T7: Adubação no substrato + 1g. $\mathrm{L}^{-1}$ zinco via foliar $\left(\mathrm{ZnSO}_{4}\right)$; T8: adubação no substrato +1 g. $\mathrm{L}^{-1}$ boro $\left(\mathrm{H}_{3} \mathrm{BO}_{4}\right)+1 \mathrm{~g}$. $\mathrm{L}^{-1}$ zinco $\left(\mathrm{ZnSO}_{4}\right)$ via foliar. A adubação no substrato consistiu em uma aplicação de fosfato de cálcio $\left(200 \mathrm{mg} . \mathrm{dm}^{-3}\right)$ somente no pré-plantio, mais adubação básica sete dias após o plantio, e repetida a cada 30 dias, composta de: $0,31 \mathrm{~g}$. $\mathrm{L}^{-1}$ de sulfato de magnésio + $0,022 \mathrm{~g}$. $\mathrm{L}^{-1}$ de sulfato de zinco $+0,006 \mathrm{~g}$. $\mathrm{L}^{-1}$ de sulfato de cobre $+0,019 \mathrm{~g} . \mathrm{L}^{-1}$ de molibdato de amônio $+0,71 \mathrm{~g}$. $\mathrm{L}^{-1}$ de sulfato de amônia $+0,2 \mathrm{~g}$. $\mathrm{L}^{-1}$ de fosfato de cálcio. As adubações foliares foram realizadas 30 dias após o plantio e repetidas a cada 30 dias, durante 7 meses.

Tabela 1. Tratamentos utilizados para o estudo das adubações foliares de Zn e B e via substrato.

\begin{tabular}{|c|c|c|}
\hline Tratamentos & $\begin{array}{c}\text { Adubação no } \\
\text { substrato }\end{array}$ & Adubação via foliar \\
\hline 1 & - & - \\
\hline 2 & - & $\mathrm{B}$ \\
\hline 3 & - & $\mathrm{Zn}$ \\
\hline 4 & - & $\mathrm{B}+\mathrm{Zn}$ \\
\hline 5 & + & - \\
\hline 6 & + & $\mathrm{B}$ \\
\hline 7 & + & $\mathrm{Zn}$ \\
\hline 8 & + & $\mathrm{B}+\mathrm{Zn}$ \\
\hline
\end{tabular}


Para aplicação das adubações foliares, foi utilizado pulverizador manual com um volume de solução de $1 \mathrm{~L}$, suficiente para molhar a superfície das folhas, sem que houvesse escorrimento da solução. Utilizaram-se 2 vasos por parcela com uma planta em cada, totalizando 64 plantas. $\mathrm{O}$ experimento foi mantido em casa de vegetação, com Sombrite ${ }^{\circledR} 50 \%$, sob nebulização intermitente para manutenção da umidade.

As mudas foram avaliadas após sete meses de cultivo, observando-se altura das plantas, número de folhas por planta, largura das folhas e diâmetro médio das rosetas. Para a medição da altura de plantas usou-se régua graduada em milímetros, colocada a partir da base até o ponto extremo encontrado na planta, com resultado em centímetros. Além disso, foram contadas as folhas completamente expandidas; para determinação da largura das folhas mediu-se sua parte mediana; e para o cálculo do diâmetro médio da roseta, foi utilizado paquímetro graduado em milímetros.

Os dados experimentais foram submetidos a análises de variância e teste de médias com o auxílio do programa estatístico SISVAR (FERREIRA, 1999). As médias foram comparadas pelo teste de Skott Knott $(\mathrm{P} \leq 0,05)$.

\section{RESULTADOS E DISCUSSÃO}

As plantas que mais cresceram foram as que não receberam adubação foliar, no substrato ou que receberam apenas zinco, como pode ser observado na figura 1.

As plantas com menor desenvolvimento foram as cultivadas em substrato com adubo e as que receberam adubação foliar de boro e zinco associados.

Quando se utilizou a adubação no substrato, os maiores valores de altura de plantas foram observados com a aplicação de Zn e B separadamente (figura 1). O zinco atua como co-fator enzimático; é essencial para a atividade, regulação e estabilização da estrutura proteica; participa na ativação enzimática da trifosfato-desidrogenase, enzima essencial na glicólise, bem como nos processos de respiração e fermentação. Esse micronutriente catiônico também afeta a síntese e conservação de auxinas, hormônios vegetais envolvidos no crescimento (DECHEN \& NACHTIGALL, 2006). Observa-se que, mesmo com a aplicação de sulfato de zinco via substrato, o fornecimento de $\mathrm{Zn}$ via foliar favoreceu o desenvolvimento em altura das plantas (figura 1).

Além disso, independentemente da adubação realizada via substrato, com a aplicação de B via foliar, foram constatadas alturas semelhantes nas plantas, porém inferiores às observadas nas cultivadas no tratamento-controle (figura 1). Em relação à presença de boro nas plantas, esse nutriente tem importante função na translocação de açúcares, no metabolismo de carboidrato e nitrogênio, e na atividade de hormônios; intervém na absorção de água e no metabolismo de glicídios e dos cátions, principalmente do $\mathrm{Ca}$, na formação da pectina de membranas celulares; tem também influência no metabolismo e transporte de carboidratos (DECHEN \& NACHTIGALL, 2006).

Em relação ao número de folhas formadas, não houve interação entre a adubação via substrato e via foliar. Na fi- gura 2, observa-se que a ausência de adubação foliar representou, em média, maior número de folhas por planta. Ao contrário, menor número de folhas foi observado nas plantas que receberam boro via foliar, conforme se vê também na figura 2. Esse resultado foi semelhante aos observados para altura média das plantas (figura 1): aquelas que não receberam adubação foliar também cresceram mais.

Uma característica marcante dos micronutrientes, principalmente do boro, é que o limite entre a essencialidade e a toxidez de suas doses para as plantas é muito tênue. Assim sendo, um aspecto importante para a produção de mudas de bromélias diz respeito à definição da quantidade de boro a ser aplicada (DECHEN \& NACHTIGALL, 2006).

A aplicação da adubação foliar composta apenas por zinco proporcionou a formação de maior número de folhas, quando comparada à desse nutriente associado ao boro, indicando o efeito negativo desse último. Observaram-se nas plantas sintomas de toxidez ocasionada pela aplicação de boro. Pela figura 3, pode-se constatar necrose na ponta das folhas, além de menor desenvolvimento das plantas. Segundo DECHEN \& NACHTIGALL (2006), a toxidez de boro é observada nas folhas pela ocorrência de amarelecimento, que se estende para as margens, progredindo para necroses. Esse efeito em bromélias já havia sido sugerido por alguns viveiristas, mas ainda não há registros técnicos sobre esse sintoma.

Em relação à largura das folhas formadas, também não se observou interação entre a adubação via substrato e foliar. A aplicação foliar de B associado ao $\mathrm{Zn}$ proporcionou a formação de folhas mais estreitas em relação aos demais tratamentos (figura 4). Maior largura das folhas foi observada quando não se realizou adubação no substrato, (figura 5), com a produção de plantas mais desenvolvidas, conforme se vê na figura $5 b$.

Em relação ao diâmetro da roseta, também foi verificado que ficaram maiores na ausência de adubação de substrato (Figura 6). Não houve efeito da adubação foliar sobre esse parâmetro.

RODRIGUES (2004) relata bom desenvolvimento das mudas de bromélia imperial com maiores diâmetros de base e de roseta, altura e número de folhas pela aplicação da solução de Hoagland (1950) na fase de aclimatização. Nessa fase de desenvolvimento das mudas, a aplicação de adubação não se mostrou eficiente, mas foi possível verificar e comprovar o efeito fitotóxico do uso de Boro e Zinco, fato esse já indicado por alguns viveiristas.

No trabalho realizado, o fornecimento de boro e zinco não teve efeito promotor de crescimento nessa espécie. Observou-se ainda, aos 90 dias, que a aplicação de boro provocou queimaduras e necrose na ponta das folhas das bromélias, prejudicando seu desenvolvimento.

\section{CONCLUSÕES}

Nas condições em que o experimento foi conduzido, pode-se concluir que:

1. Não foram produzidos resultados satisfatórios de de- 
senvolvimento nas mudas de bromélia imperial em resposta à aplicação de adubação via substrato ou foliar;

2. A adubação foliar com boro provocou toxidez nas mudas, causando queimaduras e necrose na ponta das folhas.

3. A aplicação de zinco não ocasionou toxidez nas plantas, mas dificultou seu desenvolvimento.

\section{REFERENNCIAS}

CARVAlHO, L. F. N. Boletim da Sociedade Brasileira de Bromélias, Rio de Janeiro, v. 7, n. 3, 1997.

DECHEN, A. R. \& NACHTIGALL, G. R. Micronutrientes. In: FERNANDES, M. S. (ed.) Nutrição Mineral de Plantas. Sociedade Brasileira de Ciência do Solo, Viçosa, 2006. p.327-352

FERREIRA, C.A.; PAIVA, P. D. O.; RODRIGUES, T. M.; RAMOS, D. P.; CARVALHO, J. G. \& PAIVA, R. Desenvolvimento de mudas de bromélia (Neoregélia cruenta ( R. Eraham) L. B. Smith) cultivadas em diferentes substratos e adubação foliar. Revista Ciência e Agrotecnologia Lavras, MG, v.31, n.3, p 666-671, 2007.

FERREIRA, D.F. SISVAR. Versão 4.3 (Build 41). Lavras: UFLA/DEX, 1999. 4 disquetes.

HOAGLAND, D. R.; ARNON, D. I. The water culture method of growing plants without soil. Berkeley: University of California, 1950. 32p.

LEME, E.M.C.; MARIGO, L.C. Bromélias na Natureza, Rio de Janeiro, 1993.

MALAVOLTA, E.; Elementos da nutrição mineral de plantas. Piracicaba: CERES, 1980. 251p.

MELO, T. B. de. As bromélias no paisagismo. Bromélia, Rio de Janeiro, v.3, n.1, p.3-7, mar./1996.

PAULA, C. C. de. Cultivo de bromélias. Viçosa: Aprenda Fácil, 2000.139 p.

QUAGGIO, J. A.; PIZA JUNIOR, C. T. Micronutrientes para frutíferas tropicais. In: FERREIRA, M. E.; CRUZ, M. C. P.; RAIJ, B. van; ABREU, C. A. (ed.). Micronutrientes tóxicos e metais pesados na agricultura. Jaboticabal: CNPq/Fapesp/Potafos, 2001. p. 459-491.

RODRIGUES, T. M.; PAIVA, P. D. O.; RODRIGUES, C. R.; CARVALHO, J. G.; FERREIRA, C.A. \& PAIVA, R. Desenvolvimento de mudas de bromélia imperial (Alcantarea imperialis) em diferentes substratos. Revista Ciência e Agrotecnologia, Lavras (MG), v.28, n. 4, p 757-763, 2004.

SMITH, L.B.; DOWNS, R.J. Flora neotrópica: Bromeliaceae, New York: New York Botanical Gardens, v.14, n.2, p. 1268.1977.
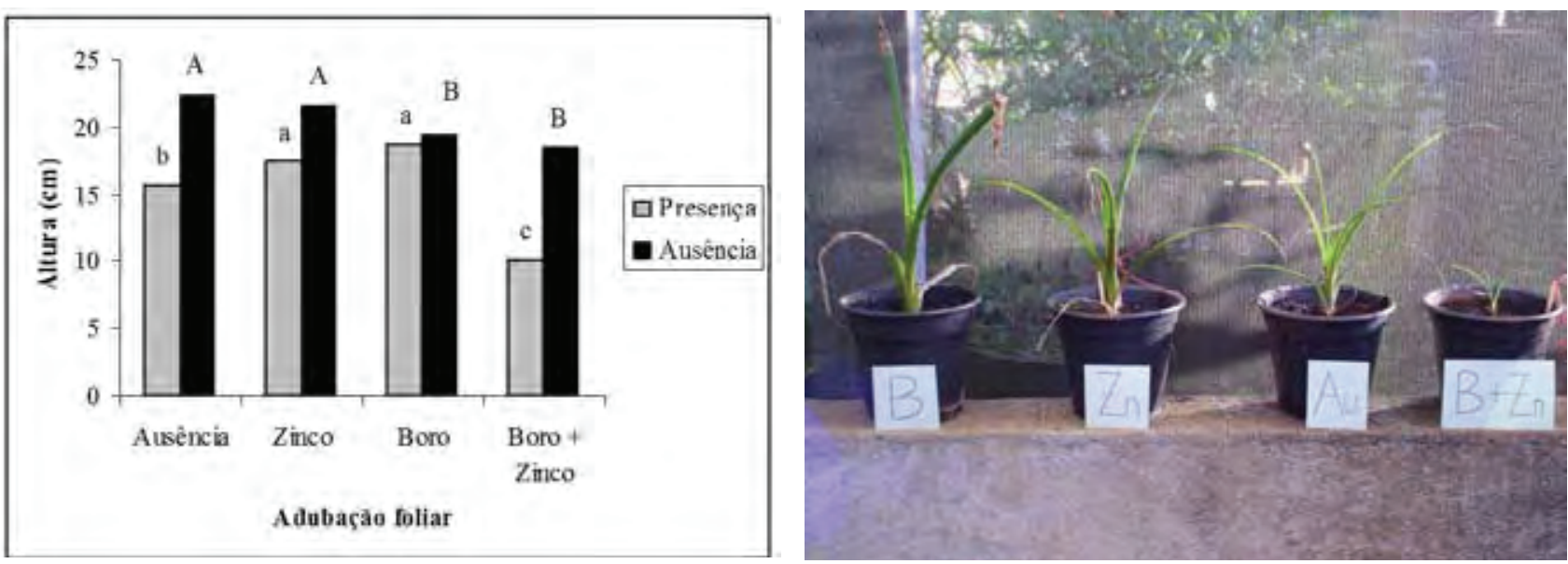

Figura 1. Altura das mudas de bromélia imperial desenvolvidas em função da aplicação de adubação foliar e no substrato. Médias seguidas de mesma letra maiúscula (sem adubação via substrato) ou mesma letra minúscula (com adubação via substrato) não diferem entre si pelo teste de Skott Knott.

Figure 1. Height of seedlings of bromeliad imperial developed according to the application of foliar fertilizer and substrat. Medium followed the same capital letter (without fertilization) or the same minute latter (with fertilization) not differ in the test Skott Knott. 


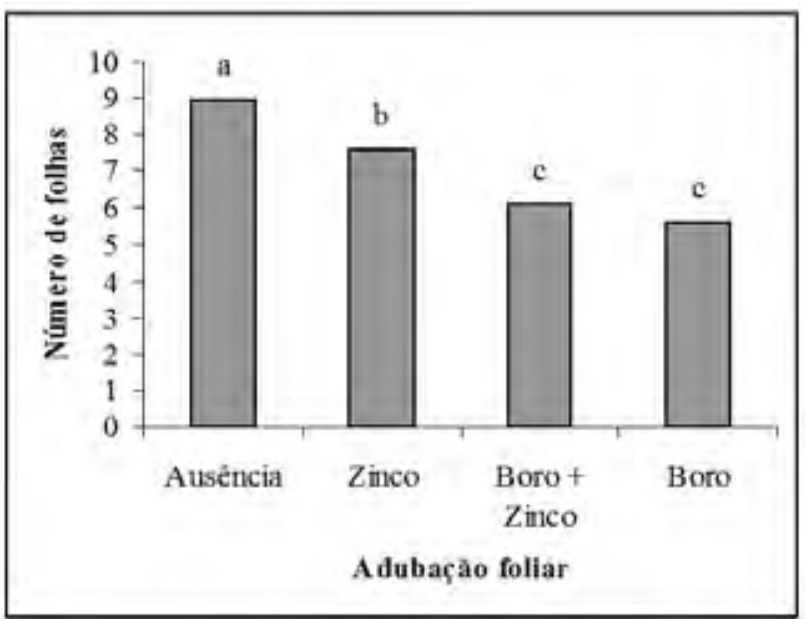

Figura 2. Número de folhas formadas em mudas de bromélia imperial em função da aplicação de adubação foliar. Médias seguidas de mesma letra não diferem entre si pelo teste de Skott Knott.

Figure 2. Number of leaves formed in seedlings of bromeliad imperial according to the application of foliar fertilizer. Medium followed the same letter do not differ in the test Skott Knott.
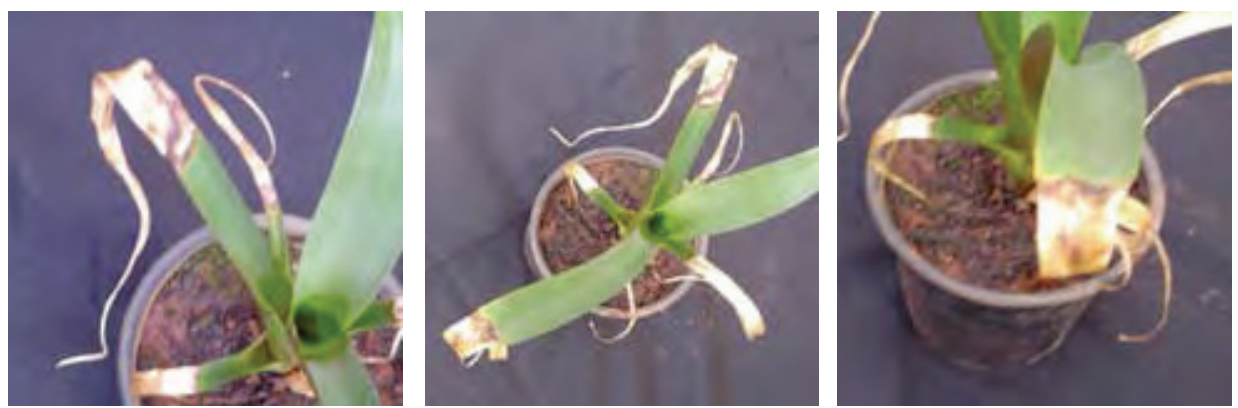

Figura 3. Injúrias ocasionadas pela aplicação foliar de boro em mudas de bromélia imperial aos 90 dias de cultivo.

Figure 3. Injury caused by foliar application of boron in the imperial bromeliad seedlings of 90 days of cultivation.

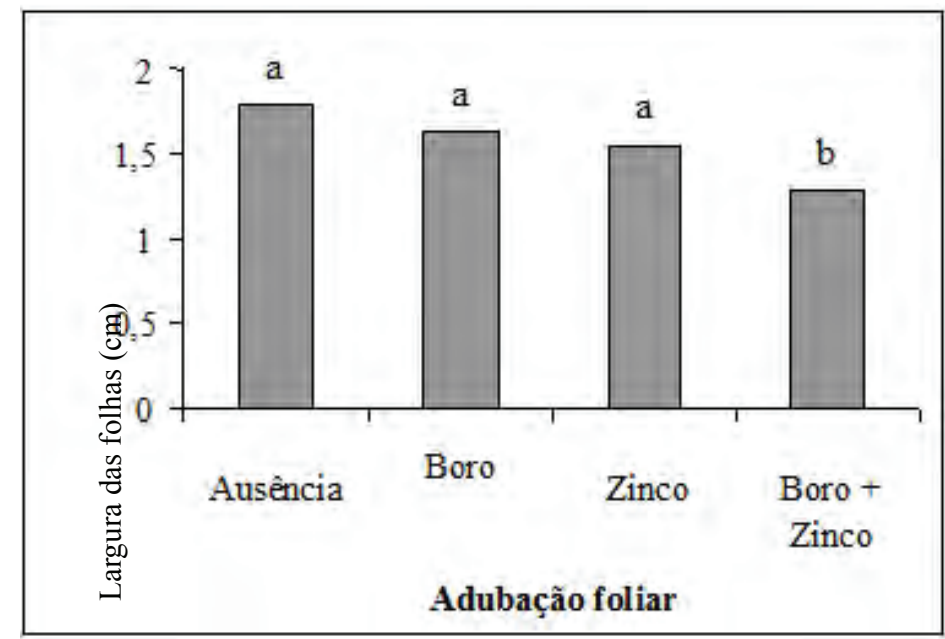

Figura 4. Largura das folhas (cm) formadas em mudas de bromélia imperial em função da aplicação da adubação foliar. Médias seguidas de mesma letra não diferem entre si pelo teste de Skott Knott.

Figure 4. Width of the leaves (cm) formed in seedlings of bromeliad imperial according to the application of foliar fertilizer. Medium followed the same letter do not differ in the test Skott Knott. 

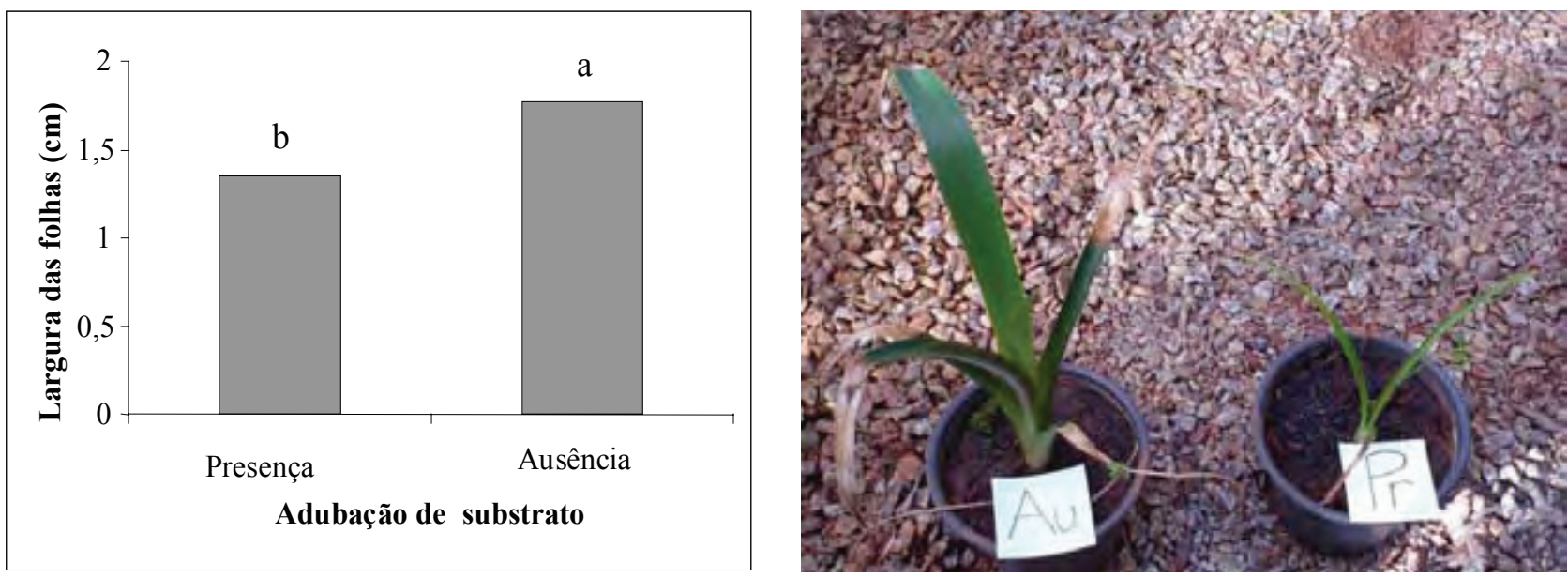

Figura 5. Largura das folhas $(\mathrm{cm})$ formadas em mudas de bromélia imperial em função da aplicação da adubação de substrato. Médias seguidas de mesma letra não diferem entre si pelo teste de Skott Knott.

Figure 5. Width of the leaves (cm) formed in seedlings of bromeliad imperial according to the application of fertilization of substrate. Medium followed the same letter do not differ in the test Skott Knott.

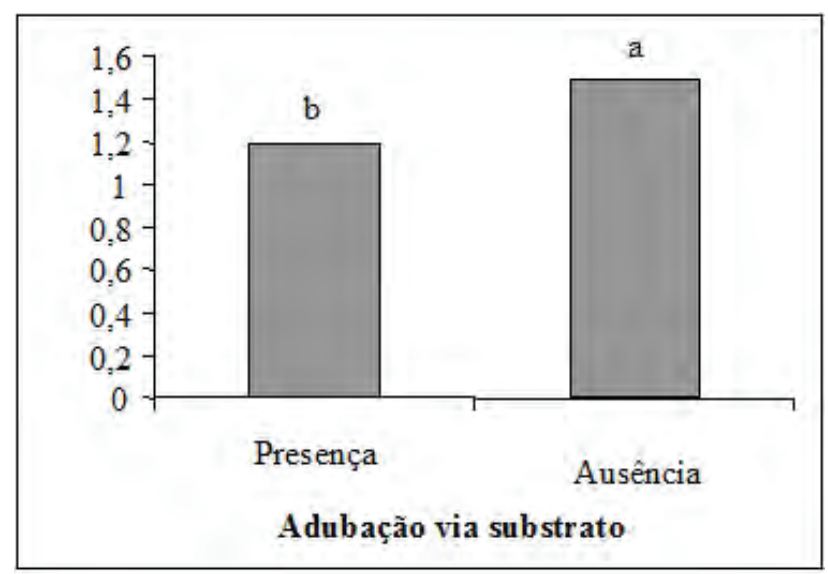

Figura 6. Diâmetro da roseta $(\mathrm{cm})$ de mudas de bromélia imperial desenvolvidas em função da aplicação de adubação no substrato. Médias seguidas de mesma letra não diferem entre si pelo teste de Skott Knott.

Figure 6. Diameter of the rosette (cm) of seedlings of bromeliad imperial developed according to the application of fertilizer in the substrate. Medium followed the same letter do not differ in the test Skott Knott. 